\title{
Dynamic expression of smRNA from fecal exosome in disease progression of an inflammatory bowel disorder mouse model
}

\author{
Sean Manning ${ }^{1 \#} \wedge$, Shisui Torii ${ }^{2,3 \#}$, Hannah M. Atkins ${ }^{1 \wedge}$, Yuka Imamura Kawasawa ${ }^{2,3,4} \wedge$ \\ ${ }^{1}$ Department of Comparative Medicine, Penn State University College of Medicine, Hershey, PA, USA; ${ }^{2}$ Institute for Personalized Medicine, \\ Department of Biochemistry and Molecular Biology, Penn State University College of Medicine, Hershey, PA, USA; ${ }^{3}$ Animal Genome Institute, \\ Palmyra, PA, USA; ${ }^{4}$ Department of Pharmacology, Penn State University College of Medicine, Hershey, PA, USA \\ Contributions: (I) Conception and design: S Manning, YI Kawasawa; (II) Administrative support: None; (III) Provision of study materials or patients: \\ S Manning, YI Kawasawa; (IV) Collection and assembly of data: S Manning, HM Atkins, YI Kawasawa; (V) Data analysis and interpretation: All \\ authors; (VI) Manuscript writing: All authors; (VII) Final approval of manuscript: All authors. \\ "These authors contributed equally to this work. \\ Correspondence to: Yuka Imamura Kawasawa, PhD. Penn State University College of Medicine, 500 University Dr. Mail Code H093, Hershey, PA \\ 17033, USA. Email: yimamura@pennstatehealth.psu.edu.
}

Background: Inflammatory bowel disease (IBD) is a chronic inflammatory disorder of the gastrointestinal (GI) tract affecting over 3 million adults in the United States. Despite being widespread, reliable early diagnostic tests are not available.

Methods: We examined exosomal small RNA (smRNA), specifically targeting microRNA (miRNA) and piRNA from the stool samples of IBD model mice, interleukin 10 knockout mice (IL-10 KO), as a potential diagnostic marker. Stool samples were specifically chosen because they are readily available, and collection is noninvasive. A modified Crohn's disease activity index was used to score each mouse's clinical condition twice a week. At the end of the experimental period, the GI tract was collected, and disease severity was scored.

Results: Histopathology showed a significant increase in inflammation and proliferation within the proximal and distal large intestines. smRNA profiles were examined upon conventional housing (startpoint) which is a determinant factor of spontaneous IBD progression in the IL-10 KO mice, terminal illness (end-point), and 6 weeks before the end-point (mid-point), when the mice were still phenotypically healthy. We found 504 smRNA that were significantly differentially expressed between before symptom onset and terminal sedation. These changes were not detected in wild-type samples. Moreover, clustering analysis of expression changes over the disease progression identified a unique set of smRNAs that primarily target pro-inflammatory or anti-inflammatory genes. The expression of smRNAs that suppresses proinflammatory genes was higher at 6 weeks before terminal sedation, suggesting the downregulation of the pro-inflammatory genes advances the terminal illness of the IBD.

Conclusions: Our study proposes that fecal exosomal smRNA profiling offers a new opportunity to monitor the inflammatory status of the gut with a capability of detecting its pro-inflammatory (asymptomatic) status. Our next step is to understand spatiotemporal interplay of these exosomes and the host cells in the gut as well as the complete biochemical makeup of fecal exosomes, such as mRNA, DNA, protein, and lipids. This study paves the path to the future utilization of fecal exosome in early diagnosis, follow-up, and potential mitigation of the chronic gut inflammation.

Keywords: Inflammatory bowel disease (IBD), IL-10 knockout mouse, smRNA, fecal exosome

Received: 23 February 2021; Accepted: 05 July 2021; Published: 30 July 2021.

doi: 10.21037/exrna-21-8

View this article at: https://dx.doi.org/10.21037/exrna-21-8

^ ORCID: Sean Manning, 0000-0002-8190-1104; Hannah M. Atkins, 0000-0001-7689-1477; Yuka Imamura Kawasawa, 0000-0002-8638-6738. 


\section{Introduction}

Inflammatory bowel disease (IBD), commonly categorized into either Crohn's disease (CD) or ulcerative colitis (UC), is a common cause of chronic inflammation in the gastrointestinal (GI) tract (1). While often referred to under the same umbrella term IBD, CD and UC have several differences. Patients with CD often have patches of disease through the whole GI tract, with inflammation affecting the full thickness of the intestine. In contrast, UC manifests mostly in the colon and rectum with inflammation often limited to the mucosa (2). Clinical signs usually consisting of diarrhea, fever, and abdominal pain are often relapsingremitting, with flare-ups followed by remission periods (3). In all cases, IBD leads to a low quality of life, often with multiple hospitalizations and surgical procedures (4).

While over 3 million adults in the US are affected, diagnosis is often prolonged (5). According to the European Crohn's and Colitis Organization [ECCO], "a single reference standard for the diagnosis of Crohn's disease [CD] or ulcerative colitis [UC] does not exist" (6). Current methods for diagnosing IBD include a combination of physical exams, blood tests, stool examinations, endoscopy, biopsies, and imaging (7). This battery of tests results in an average 2-3-month delay in diagnosis for UC and 6-7-month delay for CD $(8,9)$. More recently, the study of exosomes and the exosomal small RNA (smRNA) they contain have shown to be a valuable diagnostic marker in IBD and many other diseases $(10,11)$.

Exosomes are a sub-type of extracellular nano-sized vesicle that have been shown to mediate extracellular communication via the transport of various biomolecules. Exosomes transport coding and non-coding RNA, including smRNA. This signaling process impacts several physiological and pathological pathways by modulation of protein expression. Due to their involvement in critical biological functions, studies into the use of exosomal smRNA as biomarkers in various diseases and tissues have grown significantly (12-14). For example, smRNA show promise as a diagnostic test, differentiating colitis subtypes, and evaluating therapy response (15). While studied extensively in serum, intestinal biopsies, and even saliva $(2,15)$, little is known about fecal exosomes' role in disease development. In contrast to serum, feces are abundant, GI tract-specific, and noninvasive to collect. Studies such as Koga et al. (16) demonstrating the stability of smRNA in fecal exosomes, and Liu et al. (17) investigating fecal smRNA and the microbiome, show value in the use of feces to evaluate smRNA. However, smRNA in exosomes in IBD have not been studied.

Our study evaluated fecal smRNA profiles and tracked changes before and after IBD development in IL-10 KO mice and compared them to wild-type mice. IL-10 KO mice have been extensively studied as an IBD model (18-22). Although IL-10 KO mice are viable and fertile when housed under specific pathogen free (SPF) conditions, once they are conventionally housed, they develop altered lymphocyte and myeloid profiles, elevated serum amyloid A levels, altered responses to inflammatory or autoimmune stimuli, increased prevalence of colorectal adenocarcinoma, and spontaneous development of chronic enterocolitis (23). While spontaneous enterocolitis is the target of this study, human IBD patients can also similarly develop secondary systemic amyloidosis (SSA) (24) are prone to the development of colorectal adenocarcinoma (25), and have dysfunctional immune responses (1). We hypothesize that fecal exosomal smRNA shows a unique profile in disease progression profile in the IL-10 KO mice and monitored the smRNA transcriptome over 30 weeks while the animals spontaneously develop symptoms. We propose this unique smRNA fingerprint is a promising, noninvasive diagnostic tool for IBD.

We present the following article in accordance with the ARRIVE checklist (available at https://dx.doi.org/10.21037/ exrna-21-8).

\section{Methods}

\section{Animals}

Fourteen each of four-week-old IL-10 KO mice (Stock No. 002251 with C57BL/6J background, IMSR Cat\# JAX:002251, RRID:IMSR_JAX:002251) and control wild-type (WT) mice (C57BL/6J, MGI Cat\# 5657312, RRID:MGI:5657312, equal number of females and males (seven each in each genotype) was used) were obtained from The Jackson Laboratories (Bar Harbor, Maine). Each mouse was then housed conventionally and individually in polycarbonate cages, static, wire-top cages on corncob bedding (7092 Harlan Teklad, Madison, WI), and had ad lib access to irradiated rodent chow (2918, Harlan Teklad, Madison, WI) and tap water. The animal facility was programmed with a 12:12-h light: dark cycle. Room temperature was maintained at $20 \pm 2{ }^{\circ} \mathrm{C}$, and air humidity ranged between $30 \%$ to $60 \%$. Environmental enrichment was provided in the form of an Enviropak (Lab Supply, Fort Worth, TX). Mice were free of ectromelia virus, 
Table 1 Modified Crohn's disease activity index

\begin{tabular}{ll}
\hline I. Excreted perianal mucous & 0 or 1 \\
II. Rectal prolapse & 0 or 1 \\
III. Diarrhea & 0 or 1 \\
IV. Weight loss $>5 \%$ & 0 or 1 \\
\hline
\end{tabular}

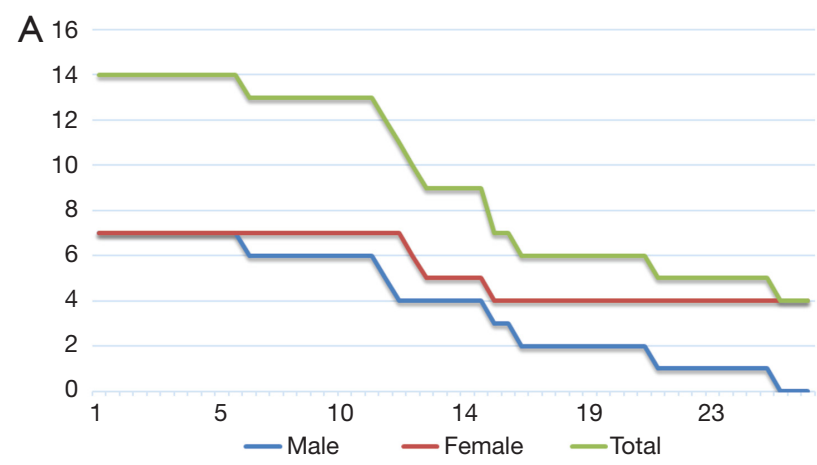

B

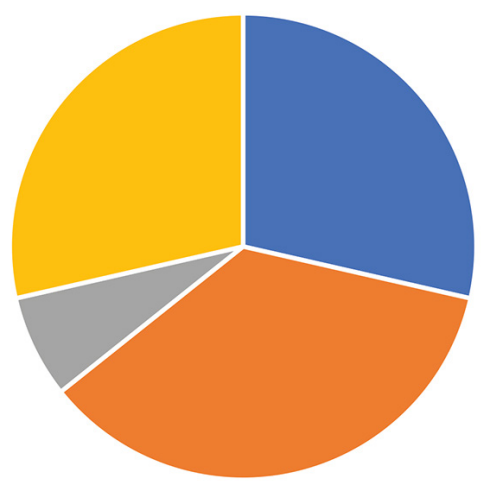

mFound near death/dead $\quad=>25 \%$ weight loss
$=$ Score 3,3 consecutive times $\quad$ No disease

Figure 1 Survival curves for IL-10 KO mice. (A) The number of survivors at the indicated weeks is shown for IL-10 KO. The number of mice euthanized before achieving humane endpoints was counted. (B) The pie chart shows the proportion of the cause of death in IL-10 KO mice.

Theiler's encephalomyelitis virus, Hantaan virus, $\mathrm{K}$ virus, lactate dehydrogenase elevating virus, lymphocytic choriomeningitis virus, mouse adenovirus types 1 and 2, mouse cytomegalovirus, mouse hepatitis virus, mouse minute virus, murine norovirus, mouse parvovirus, mouse thymic virus, pneumonia virus of mice, polyoma virus, Reovirus 3, Rotavirus, encephalomyelitis virus, Sendai virus, Bordetella bronchiseptica, Flavum rodentium, Citrobacter rodentium, Clostridium piliforme, Corynebacterium kutscheri Corynebacterium bovis, Helicobacter spp., Mycoplasma pulmonis, Pasteurella spp., Salmonella spp., Streptobacillus moniliformis, Klebsiella pneumonia, Pneumocystis murina, Pseudomonas spp., Staphylococcus aureus, Streptococcus pneumonia, $\beta$-hemolytic Streptococcus spp., endo-and ectoparasites, enteric protozoa, and Encephalitozoon cuniculi. All experiments were conducted in accordance with institutional guidelines, the Guide for the Care and Use of Laboratory Animals (Institute for Laboratory Animal Research, 2011), and approved by the Penn State College of Medicine Institutional Animal Care and Use Committee (ID: PROTO201800170).

\section{Fecal collection and disease scoring}

The feces were collected once a week, in the morning, and stored at $-80^{\circ} \mathrm{C}$ until processing. Mice were scuffed, and a $1.5 \mathrm{~mL}$ Eppendorf tube (Fisher Scientific, Waltham, MA) was placed under the anus to collect 3-5 fecal pellets. If sufficient feces were not collected after 30 seconds, the mouse was placed in a clean cage without beading until at least 3 pellets were obtained. During this time, the mice were also scored for disease severity using a modified Crohn's Disease Activity Index (CDAI) based on Scheinin et al. (22) (Table 1). Collection continued until mice either reached a score of 3 for three consecutive times or a score of 4 , or showed rapid weight loss beyond $25 \%$, which were established as experiment end-points. The fecal samples collected at the first week of conventional housing and 6 weeks before the experimental end-point were designated as start-point samples and mid-point samples, respectively (Figure 1A). Some KO mice never developed IBD (scored 0 (no signs of disease) for the entire experimental period) and were euthanized after 30 weeks of conventional housing (Figure 1A). For WT mice (no signs of disease throughout the 30 weeks of conventional housing), we collected the feces samples starting at the average age of disease onset in $\mathrm{KO}$ mice ( 5 months) for a duration of 6 weeks. At the time of euthanasia, the GI tract was collected. The small and large intestines were separated just proximal to the cecum and fixed in $10 \%$ neutral buffered formalin for 24 hours before being transferred to $70 \%$ ethanol. The tissues were paraffin-embedded using an automated embedding machine and sectioned 5-um thick for routine Harris hematoxylin and eosin (HE) staining. The intestines were microscopically evaluated and scored by a boardcertified veterinary pathologist blinded to the groups. Five randomly-selected, $20 \times$ fields of small or large intestine 
Table 2 Disease scoring scheme for large intestinal tissues. Evaluation criteria for the large intestine for location and type of inflammatory cell infiltrates, as well as severity of epithelial changes. Scores were averaged across $5 \mathrm{X}, 20 \mathrm{X}$ objective fields for each criterion. The total mean score was also determined for each section of intestine.

\begin{tabular}{|c|c|c|c|c|c|c|c|}
\hline \multicolumn{4}{|c|}{ Inflammatory cell infiltrate } & \multicolumn{4}{|c|}{ Epithelial changes } \\
\hline \multicolumn{2}{|c|}{ Location } & \multicolumn{2}{|c|}{ Type } & \multicolumn{2}{|c|}{ Proliferation } & \multicolumn{2}{|c|}{ Necrosis } \\
\hline 0 & None & 0 & None & 0 & None & 0 & None \\
\hline 1 & Mucosa only & 1 & $\begin{array}{l}\text { Lymphocytic or } \\
\text { plasmacytic }\end{array}$ & 1 & Minimal hyperplasia & 1 & Epithelial degeneration \\
\hline 2 & $\begin{array}{l}\text { Mucosa with segmental } \\
\text { submucosa, +/- crypt epithelium }\end{array}$ & 2 & Neutrophilic & 2 & $\begin{array}{l}\text { Mild hyperplasia, mild goblet cell } \\
\text { loss }\end{array}$ & 2 & Erosions \\
\hline 3 & $\begin{array}{l}\text { Mucosa and submucosa, crypt } \\
\text { epithelium, crypt abscesses }\end{array}$ & 3 & Macrophagic & 3 & $\begin{array}{l}\text { Moderate hyperplasia, moderate } \\
\text { goblet cell loss }\end{array}$ & 3 & Ulceration \\
\hline 4 & $\begin{array}{l}\text { Transmural, multiple crypt } \\
\text { abscesses }\end{array}$ & & & 4 & $\begin{array}{l}\text { Marked hyperplasia, marked } \\
\text { goblet cell loss, some atypia }\end{array}$ & 4 & $\begin{array}{l}\text { Regionally extensive } \\
\text { ulcerations }\end{array}$ \\
\hline
\end{tabular}

Table 3 Disease scoring scheme for small intestinal tissues. Evaluation criteria for the small intestine for location and type of inflammatory cell infiltrates, as well as severity of epithelial changes Scores were averaged across $5 \mathrm{X}, 20 \mathrm{X}$ objective fields for each criterion. The total mean score was also determined for each section of intestine.

\begin{tabular}{|c|c|c|c|c|c|c|c|}
\hline \multicolumn{4}{|c|}{ Inflammatory cell infiltrate } & \multicolumn{4}{|c|}{ Epithelial changes } \\
\hline 0 & None & 0 & None & 0 & None & 0 & None \\
\hline 1 & Mucosa only & 1 & $\begin{array}{l}\text { Lymphocytic or } \\
\text { plasmocytic }\end{array}$ & 1 & Minimal hyperplasia & 1 & Epithelial degeneration \\
\hline 3 & $\begin{array}{l}\text { Mucosa and submucosa, } \\
\text { segmental muscularis }\end{array}$ & 3 & Macrophagic & 3 & Moderate hyperplasia & 3 & Moderate villous blunting \\
\hline 4 & Transmural & & & 4 & Marked hyperplasia & 4 & Villous atrophy, branched crypts \\
\hline
\end{tabular}

were scored for degree of hyperplasia, inflammation severity, goblet cell loss, and epithelial cell erosion or ulceration. Each microscopic characteristic was assigned a severity score from 0-4, based on Erben et al. (26) (Tables 2,3). The $20 x$ fields were averaged and a total severity score was calculated for each intestine region (proximal and distal small intestine, cecum, and proximal and distal large intestine).

\section{Exosomal RNA extraction}

Exosomal RNA was extracted and sequenced as follows from start-point (S), mid-point (M; 6 weeks prior to the experimental end-point), and experimental end-point (E) that is defined as either when the animals reached a score of 3 for three consecutive times or a score of 4 , or after 30 weeks if the animals did not develop clinical disease (Figure 1A). We developed a novel method to reliably extract exosomal RNA from mouse feces. Briefly, 2 fecal pellets $(\sim 60 \mathrm{mg})$ were vortexed with $0.9-2.0 \mathrm{~mm}$ stainless steel beads (Next Advance, Troy, NY) with $60 \mu \mathrm{L}$ Tris- $\mathrm{HCl}(10 \mathrm{mM})$ in a $1.5 \mathrm{~mL}$ microcentrifuge tube. Increasing amounts of Tris$\mathrm{HCl}$ until $1 \mathrm{~mL}$ total volume was reached and subsequently vortexed. The resulting mixture was then centrifuged at $10,000 \times \mathrm{g}$ for 5 minutes. The supernatant was then filtered through a $0.2 \mu \mathrm{m}$ syringe filter (ThermoFisher Scientific, Waltham, MA). Exosomes were isolated via ExoQuick TC (System Biosciences, Palo Alto, CA) according to manufacturer instructions. For quality check of exosomes, a transmission electron microscope (TEM, JEOL-1400 
transmission electron microscope (JEOL USA, Peabody, MA)) and a particle tracking system, NanoSight (NanoSight Ltd, Salisbury, UK) were used. The remainder of the purified exosomes were lysed and RNA was isolated using QuickRNA Microprep Kit (Zymo Research, Irvine, CA). The quality and quantity of the extracted RNA were determined using TapeStation RNA ScreenTape (Agilent Technologies, Santa Clara, CA) and Qubit fluorometer (ThermoFisher Scientific).

\section{Small RNA sequencing and sample quality assessment}

SmRNA-sequencing libraries were prepared from 50 to $150 \mathrm{ng}$ of total RNA using the QIAseq miRNA Library Kit (Qiagen, Germantown, MD) as per the manufacturer's instructions. This system offers a built-in Unique Molecular Identifier (UMI) application, which is used to eliminate possible PCR duplicates in sequencing datasets and therefore facilitate unbiased gene expression profiling. The unique barcode sequences were incorporated in the adaptors for multiplexed high-throughput sequencing. The final product was assessed for its size distribution and concentration using BioAnalyzer High Sensitivity DNA Kit (Agilent Technologies). Pooled libraries were diluted to $2 \mathrm{nM}$ in EB buffer (Qiagen) and then denatured using the Illumina protocol. The denatured libraries were loaded onto an SP flow cell on an Illumina NovaSeq 6000 and run for 53-71 cycles using a single-read recipe according to the manufacturer's instructions. De-multiplexed sequencing reads were generated using Illumina bcl2fastq (released version 2.20.0.422, Illumina), allowing no mismatches in the index read. Primary read mapping and UMI analysis were conducted via the GeneGlobe Data Analysis Center (Qiagen).

\section{Differential expression analysis of $s m R N A$}

Input raw read count data was used after eliminating the genes for which counts per million (CPM) of reads were lower than 1 on average. edgeR $\mathrm{R}$ package (RRID:SCR_012802) was used to normalize the raw read counts to obtain Trimmed Means of M (TMM) values for data exploratory and visualization purposes. Integrative Differential Expression Analysis for Multiple EXperiments (IDEAMEX) (27) was used for four alternative differential expression analyses, namely, edgeR, DESeq2 (RRID:SCR_015687), NOI-seq (RRID:SCR_003002), and limma-voom (RRID:SCR_010943). A multidimensional scaling (MDS) plot, a Venn diagram, and a volcano plot were also generated in IDEAMEX. An adjusted $\mathrm{P}$-value of less than 0.05 and fold change cut off at 2 were used as a significance cutoff. ComplexHeatmap R package (RRID:SCR_017270) was used to create a heatmap. Short Time-series Expression Miner (STEM, RRID:SCR_005016) (28) was used for time-course analysis. Ingenuity Pathway Analysis (IPA, Qiagen, RRID:SCR_008653) was used to analyze functional enrichment in the differentially expressed smRNA. MicroRNA Target Filter function in IPA was also used to obtain the list of predicted target genes of these smRNAs. Enrichr (RRID:SCR_001575) (29) was used to examine functional enrichment of the predicted target genes.

\section{$q R T-P C R$}

miRNA All-In-One cDNA Synthesis Kit (Applied Biological Materials, Richmond, BC, Canada) was used to convert RNA to generate. Real-time PCR amplification was performed using the PowerTrack SYBR Green Master Mix (ThermoFisher Scientific). $3 \mathrm{ng}$ of input RNA was polyA-tailed, reverse transcribed, and PCR amplified using with a 5'-primer for mmu-miR-682 (CTGCAGTCACAGTGAAGTCTG) and the universal 3' miRNA primer (Applied Biological Materials). Relative Ct values were calculated based on start-point samples and compared to the mid- (only for IL-KO) and end-point samples of the same mouse individuals. Six animals were examined for each group (IL-10 KO and wild-type) and a one-tailed paired $t$-test was used to evaluate the statistical difference. A line plot was generated using gglot2 $\mathrm{R}$ package (RRID:SCR_014601).

\section{Statistical analysis}

Histopathology statistical analysis was completed using Tukey's multiple comparisons test and Sidak's multiple comparison test (GraphPad Prism8, San Diego, CA, RRID:SCR_002798) for both the small and large intestinal sections and comparisons between sexes. Statistical significance was defined as a P-value of less than or equal to 0.05 .

\section{Results}

In IL-10 KO mice, all males $(7 / 7,100 \%)$ and only $3 / 7$ $(42.9 \%)$ of females were euthanized due to reaching experimental or humane endpoint (Figure 1A). No control 
mice were euthanized or perished by 30 weeks old (data not shown). IL-10 KO mice generally gained weight until disease onset, after which they declined. All control mice continually gained weight until the end of the experimental period. Cause of the death includes spontaneous death, significant loss of weight, or euthanization due to disease scoring more than 3 for three consecutive times. However, $\sim 30 \%$ of animals did not show any symptoms by 30 weeks old (Figure 1B).

During the necropsy of IL-10 KO mice, especially ones with high disease scores, had thickened colons with scant to no feces present compared to controls (Figure 2). The colons of IL-10 KO mice with no clinical signs appeared grossly normal for mice of their strain, sex, and age (Figure $2 A$ ). IL$10 \mathrm{KO}$ mice showed a significantly higher score in the large intestine in both the proximal and distal sections (Figure 2B). There was no significant difference between disease severity in the small intestine or cecum sections (Figure 2B). For scores broken down by category in the distal large intestine, a significantly higher score was seen for IL-10 KO mice in inflammation location and proliferation (Figure 2B). For necrosis, severity trended towards significance; however, the sample size limited the analysis (Figure 2B). The same trend was also seen in the proximal large intestine (Figure $2 B$ ). No significant differences were seen between female and male scores for either the proximal or distal large intestine (Figure 2B). Representative images of the colonic histopathology are shown in Figure 2C.

Exosomes were verified using a JEOL-1400 transmission electron microscope (Figure 3A). Additionally, the sample was run through NanoSight analysis and determined $8.89 \times 10^{10}+/-1.79 \times 10^{9}$ particles $/ \mathrm{mL}$ of exosomes in the sample ranging from $75-159 \mathrm{~nm}$ in size (Figure 3B). After RNA purification, TapeStation validated the presence of a large amount of smRNA (Figure 3C). Figure 4 also validated the quality of the smRNA-sequencing by confirming the similar levels of the gene detection between samples.

The smRNA sequencing data of start- (S) and end- (E) points detected a total of $1,472 \mathrm{smRNAs}$ that are expressed more than 1 CPM on average. Four female animals that didn't develop symptoms and samples that didn't pass our quality control were excluded from the analysis. The MDS plot shows that $\mathrm{S}$ and $\mathrm{E}$ samples manifest gross differences in smRNA profiles (Figure 5A). IDEAMEX offers a WEBbased platform to perform differential expression analyses of RNA-sequencing by four different R packages, edgeR, DESeq2, NOI-seq, and limma-voom, as well as integration of the information outcomes. The integrated Venn diagram of smRNAs that are defined as differentially expressed shows variable outcome, with only $40 \mathrm{smRNAs}$ consistently called significantly different among 4 algorithms (Figure $5 B$ and Figure S1). We selected the outcome from edgeR analysis which has the largest number (504) of smRNA for subsequent analyses (Figure 5B, C and Table S1). A heatmap is showing the top 50 differentially expressed smRNAs, with clear difference between $\mathrm{S}$ and $\mathrm{E}$ timepoints and no bias in the sex of the animals (Figure 5D).

Next, we performed the analysis to examine the change of expression throughout IBD development by using Short Time-Series Expression Miner (STEM) (28) with the datasets at the start-, mid-, and end-point. Statistically significant numbers of the smRNAs were assigned to 4 clusters (colored clusters in Figure $6 \mathrm{~A}$ and Table S1) out of total 16 clusters. Among these clusters, the purple and pink clusters show sharp contrast. Expressions of smRNA in the purple cluster remain the same at the mid-point and decrease from mid- to end-point, while those in the pink cluster increase from start- to mid-point, and then decrease from mid- to end-point. We chose miR-682 from the pink cluster and examined its expression changes in wild-type and IL-10 KO mice by quantitative reverse transcription polymerase chain reaction (qRT-PCR). We were able to reproduce a significant reduction of miR-682 in the endpoint samples while there was no change in wild-type. Mid-point samples in the $\mathrm{KO}$ mice also showed significant decrease (Figure $6 B$ ), which contradicts the upward trend in the STEM analysis (Figure $6 A$ ). Due to the limited amount of feces samples, we had to use RNA samples from different batches of preparations for the STEM analysis and qRTPCR, which could be a reason for this variability.

To understand the functional relevance of these clusters, we performed functional annotation analysis of those two clusters. The purple cluster includes such as miR-2085p, miR-367-5p, miR-342-3p, miR-4276, and miR-532$5 \mathrm{p}$ (Figure $7 A$ ). Target gene pathway analysis using the genes that are targeted by smRNA shows the enrichment of multiple pro-inflammatory pathways including the TNFalpha, KRAS and IL-2/STAT5 pathways (Figures $7 B, C$ ). The pink cluster includes three gene networks that are significantly regulated by the smRNA in this cluster, such as $\mathrm{miR}-19 \mathrm{~b}-3 \mathrm{p}, \mathrm{miR}-494-3 \mathrm{p}, \mathrm{miR}-329-3 \mathrm{p}, \mathrm{miR}-434-3 \mathrm{p}$ (Figure $8 A$ ). Both pro-inflammatory and anti-inflammatory pathways were found in the target gene pathway analysis (Figures $8 B, C$ ).

Although the number of smRNA types is small and thus not qualified as significant, one of the white (uncolored) 
A

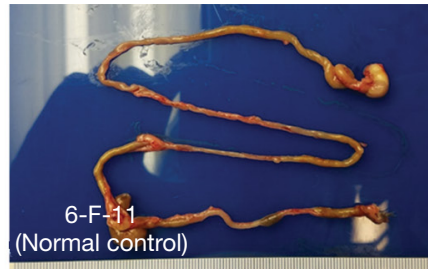

B
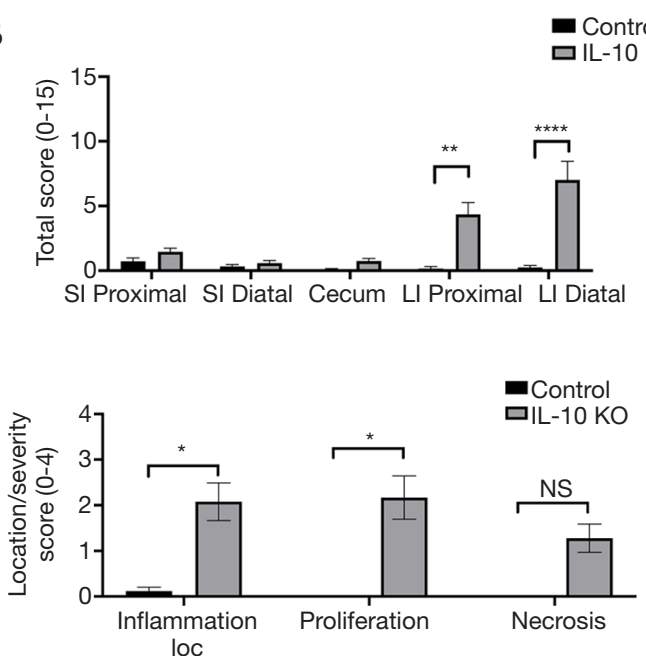

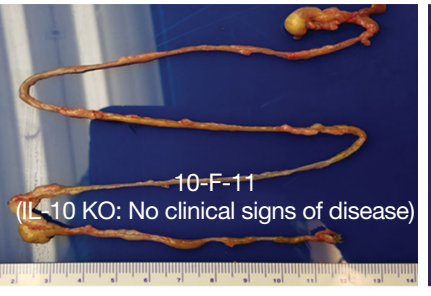

- Control

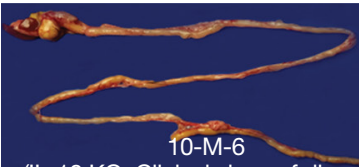

(IL-10 KO: Clinical signs of disease)

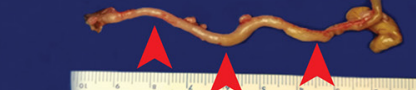

Control $\square$ Female-IL-10 KO 口Male-IL-10 KO

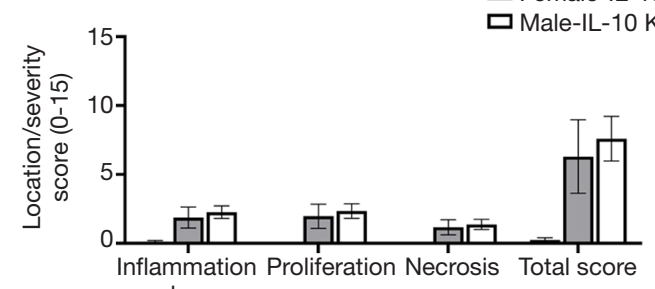

loc

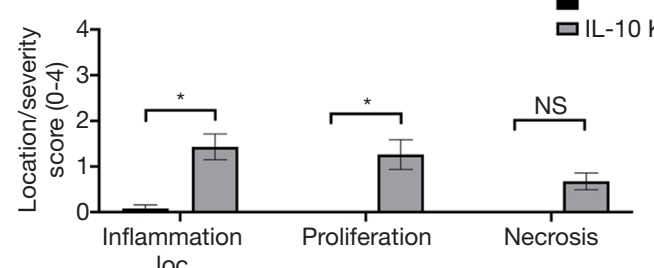

loc
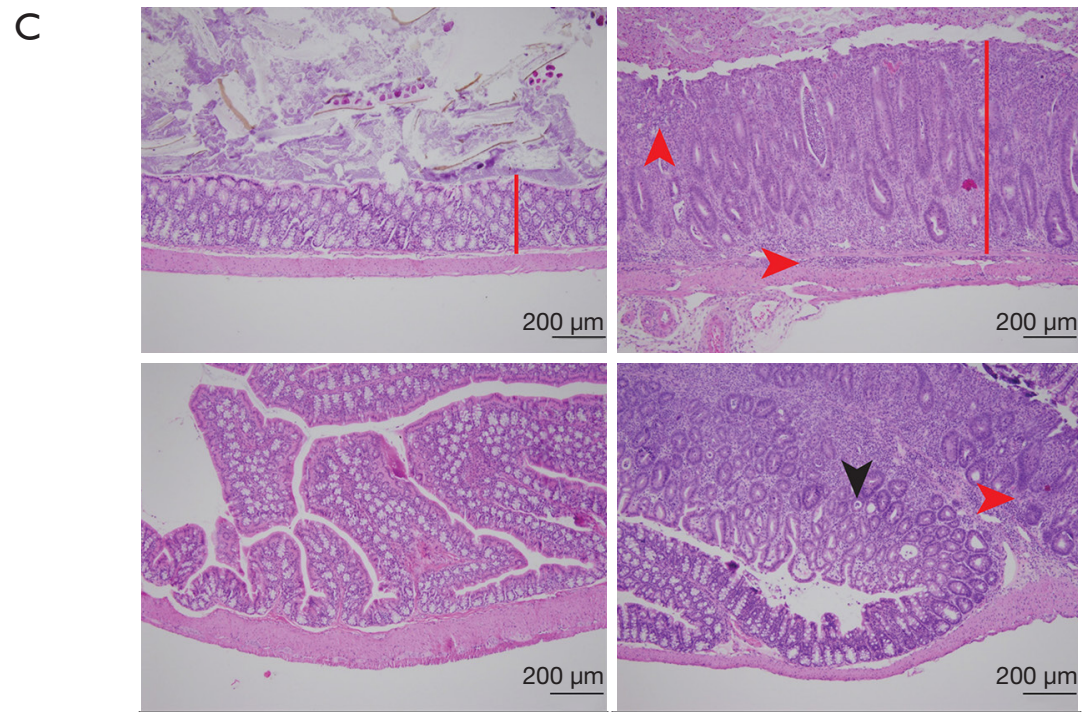

Figure 2 Pathology of the intestine in IL-10 KO mice. (A) Gastrointestinal tracts at necropsy from wild-type control (left), asymptomatic IL-10 KO (middle) and symptomatic (right) IL-10 KO mice. The large intestine from the symptomatic IL-10 KO mouse was thickened with scant to no fecal material present (red arrowhead). (B) (top left) Cumulative disease scores from four measures in indicated regions are compared between wild-type control and IL-10 KO mice. (top right) Cumulative disease scores and the scores of three measures are similar in female and male IL-10 KO that were sympomatic. Inflammation location is indicated as Inflammation Loc. (lower left and right) Comparison of the indicated measures in distal (left) and proximal (right) intestine between the indicated genotypes. *, **, and **** indicates $\mathrm{P}<0.05,<0.01$, and $<0.0001$, respectively. NS indicates comparison which does not show significant difference. (C) Hematoxylin and eosinstained wild-type control distal intestine sections (top left), clinically affected IL-10 KO (top right). Multifocal mucosal and submucosal infiltration of mixed inflammatory cells (red arrowheads) with epithelial hyperplasia (red line) and loss of goblet cells were found. Sections of the proximal large intestine of wild-type control (lower left) and affected IL-10 KO (lower right). Multifocal infiltration of mixed inflammatory cells (red arrowhead) with crypt abscessation (black arrowhead). Scale bar $=200 \mu \mathrm{m}$. 
A
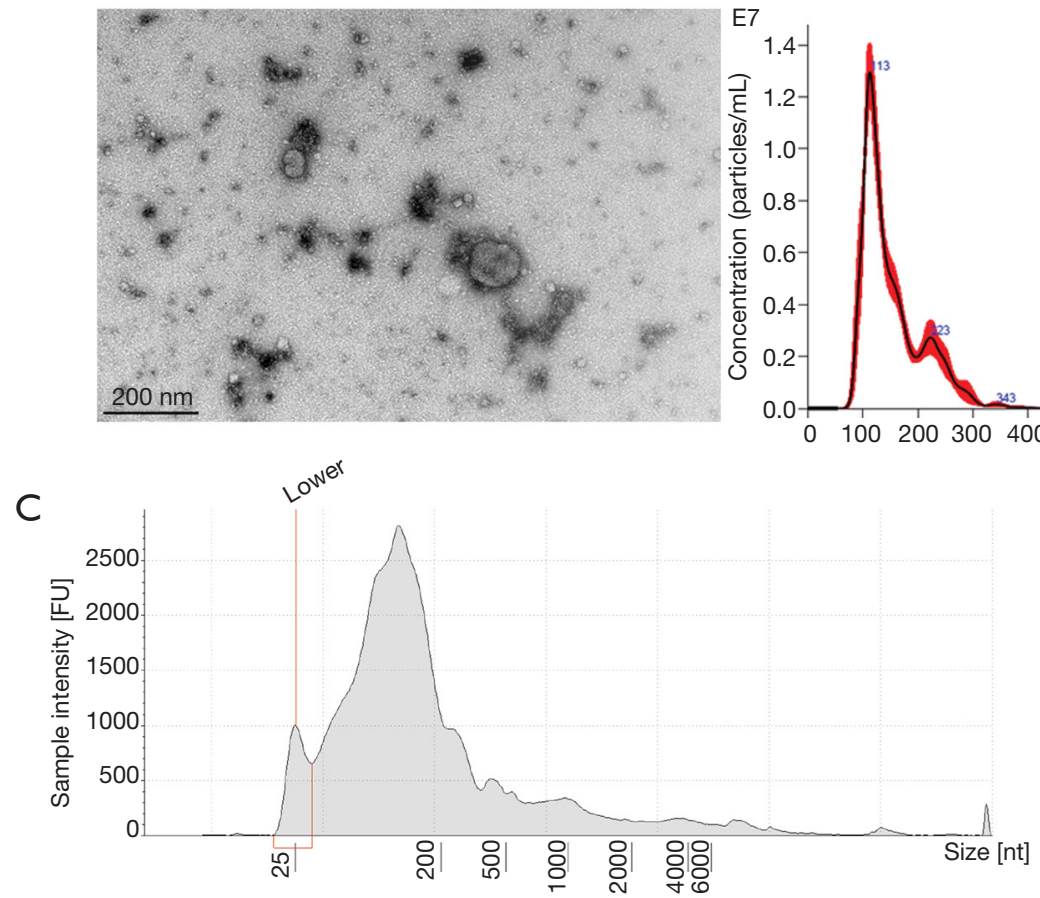

B

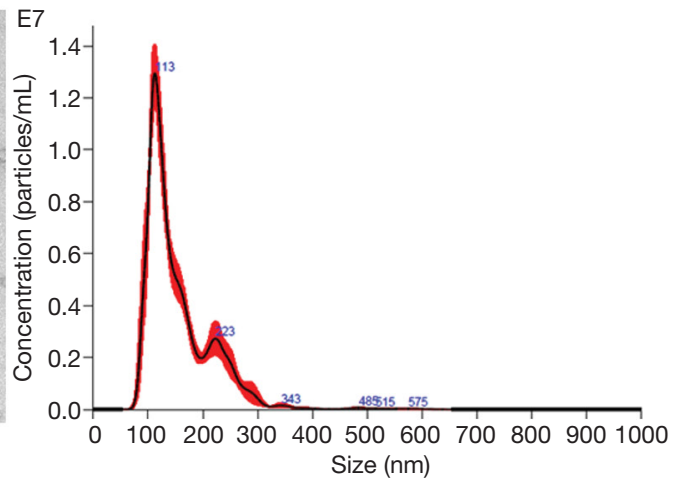

Figure 3 Extraction of exosomal smRNA from feces of mice. (A) transmission electron microscope image of exosomes taken from the feces of a wild-type mouse. (B) NanoSight analysis shows a large concentration of particles with 100 nm size, suggesting the presence of many exosomes. (C) TapeStation RNA Screen Tape shows the presence of a large amount of smRNAs.

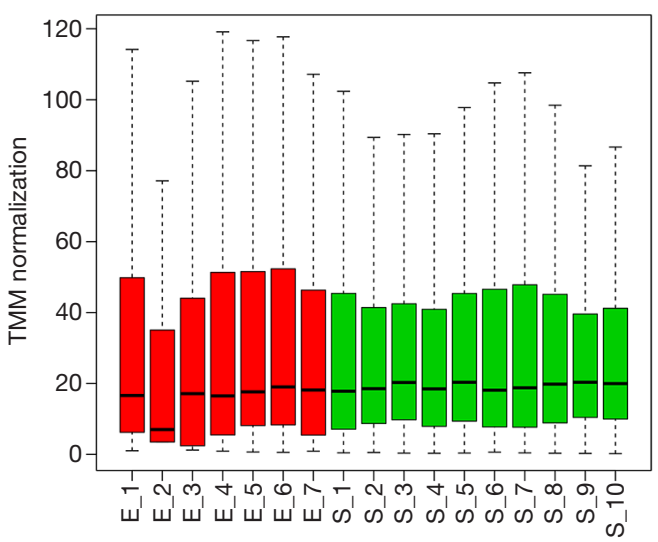

Figure 4 Quality control of smRNA sequencing. TMM normalization of RNA expression level for each sample.

clusters labelled as "cluster13" in Figure $6 \mathrm{~A}$ and Table S1 was further examined as this may work as the biomarkers of IBD progression (Figure 9). Although statistically nonsignificant, the smRNA in the "cluster13" shows the gradual increase of the expression compared with the other non-significant clusters that show a trend of the expression increase (clusters in the same row in Figure 6A). The IPA analysis did not yield a network that was controlled significantly by the smRNAs in this cluster, however, target gene pathway analysis revealed inclusion of unique pathways such as Notch signaling and the P53 pathway.

\section{Discussion}

Exosomes are a sub-type of extracellular nano-sized vesicles that mediate extracellular communication via transport of various biomolecules such as coding and non-coding RNAs (e.g., miRNA). The exosome communication process impacts several physiological and pathological pathways by modulating protein expression. Recently, investigation into the applicability of exosomal miRNA as diagnostic biomarkers has grown significantly. Here, we evaluated exosomal smRNA, specifically targeting miRNA, and piRNA from the stool samples of IBD model mice as potential markers of IBD.

In the present study, we observed an IBD-like enterocolitis in the IL-10 KO mice (Figure 2). The presence of disease in the IL-10 KO mice was confirmed on clinical signs 
A

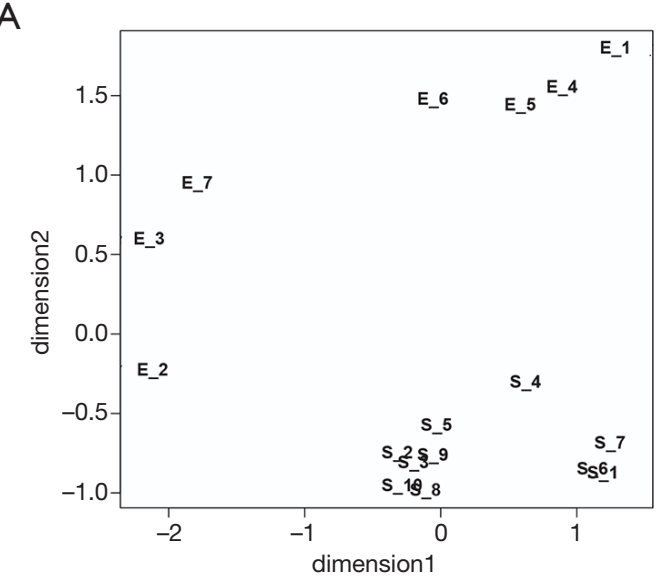

C

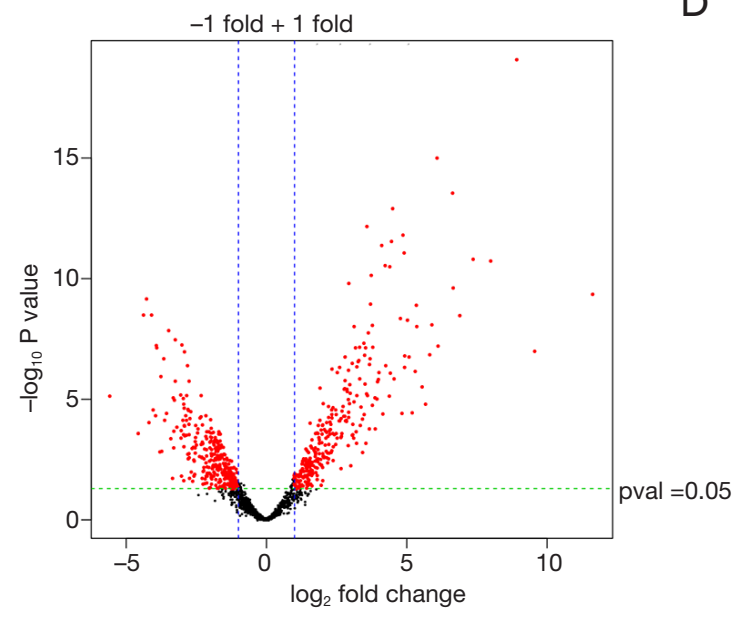

D
B

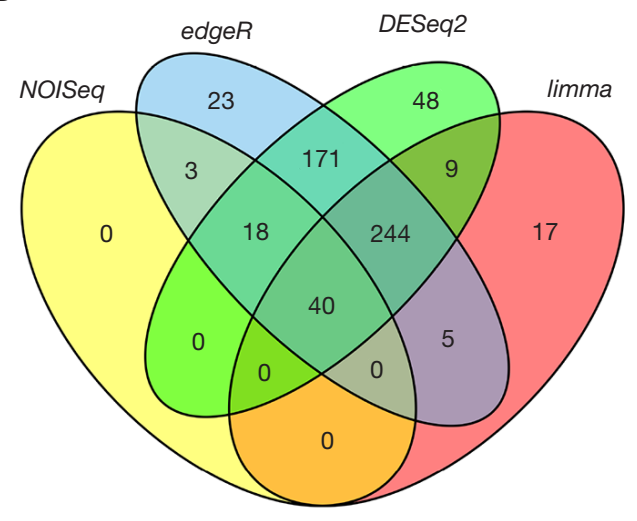

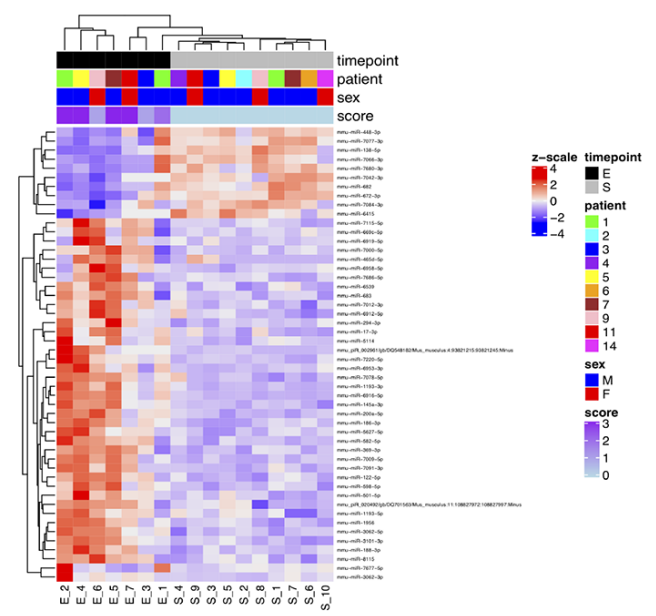

Figure 5 Differentially expressed genes between before symptom onset and terminal sedation. (A) MDS plot shows gross smRNA expression profiles are different between S (start-point) and E (end-point) samples. (B) Venn diagram of smRNAs that are defined as differentially expressed genes in edgeR, DESeq2, NOI-seq, and limma-voom. (C) The volcano plot shows the fold change and p-value of each smRNA. Red dots show genes which expressions are significantly different between S and E. (D) A clustered heatmap of the top 50 differentially expressed smRNAs is shown. The annotation bars depict sample information for its timepoint, mouse identity (patient), sex, and disease scores when feces were sampled, respectively.

and histopathology scoring. Significant differences in histopathology severity scores were identified in the large intestine between wild-type control and the IL-10 KO mice. More extensive large intestine damage than small intestine damage falls in line with previous observations (18). However, with a larger sample size, we would have likely also noted a difference in the small intestinal and cecum sections as the data was trending towards showing an increase in scores for the IL-10 KO mice.

When analyzing the scoring data, most mice developed clinical signs of disease and were euthanized by 30 weeks old. In almost all cases, this was due to the rapid weight loss beyond $25 \%$ which is considered as a humane endpoint (Figure 1), shortly after developing diarrhea and perianal mucous. However, no mice developed a score of 4 , as none of them developed rectal prolapse within the given parameters. It is possible that the IL-10 KO mice do not develop rectal prolapse as part of their disease progression. However, the weight loss may have occurred too rapidly for the disease to progress to prolapse. Of note, all 7 males eventually developed disease, while only $3 / 7$ females developed detectable disease. The differences in disease 
A
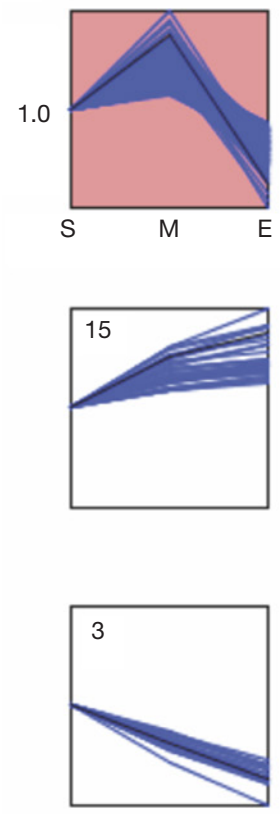
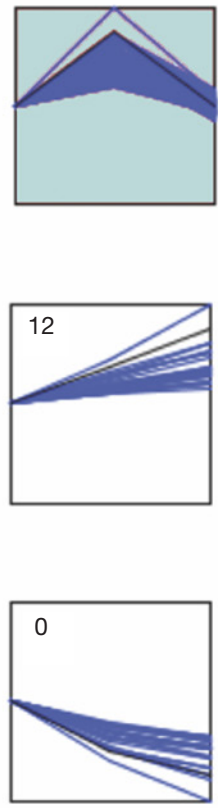

Relative expression (1.0 at S)
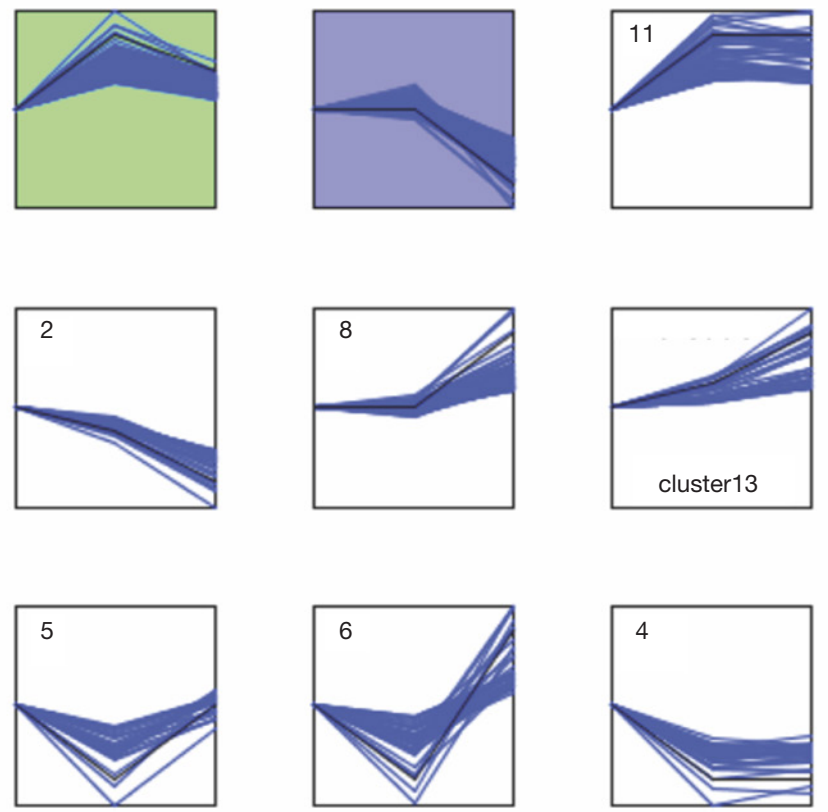

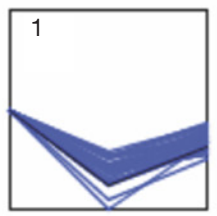

B

geno type $\rightarrow \mathrm{KO} \rightarrow \mathrm{WT}$

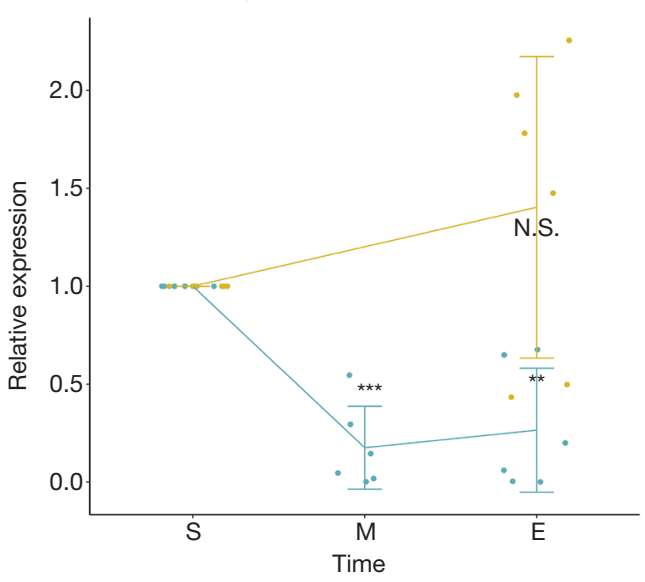

Figure 6 smRNA expression change pattern over the course of disease progression. (A) Colored clusters include a significant number of the smRNA molecules that show unique temporal changes. Blue and black lines indicate each smRNA and the average respectively. (B) Relative expression of mmu-miR-682 was validated by qRT-PCR and shown in the line plot. Error bars indicated the standard deviation of six animals chosen from both IL-10 KO (KO, light blue) and wild-type (WT). Fecal exosomal RNA was prepared from the same six mouse individuals at $\mathrm{S}$ (start-point), $\mathrm{M}$ (mid-point, $\mathrm{KO}$ animals only), and $\mathrm{E}$ (end-point). N.S. indicates no significant difference in WT samples. ** and ${ }^{* * *}$ indicate significant differences in $\mathrm{KO}$ samples compared to $\mathrm{S}$ at $\mathrm{P}<0.01$ and $<0.001$, respectively. 
A

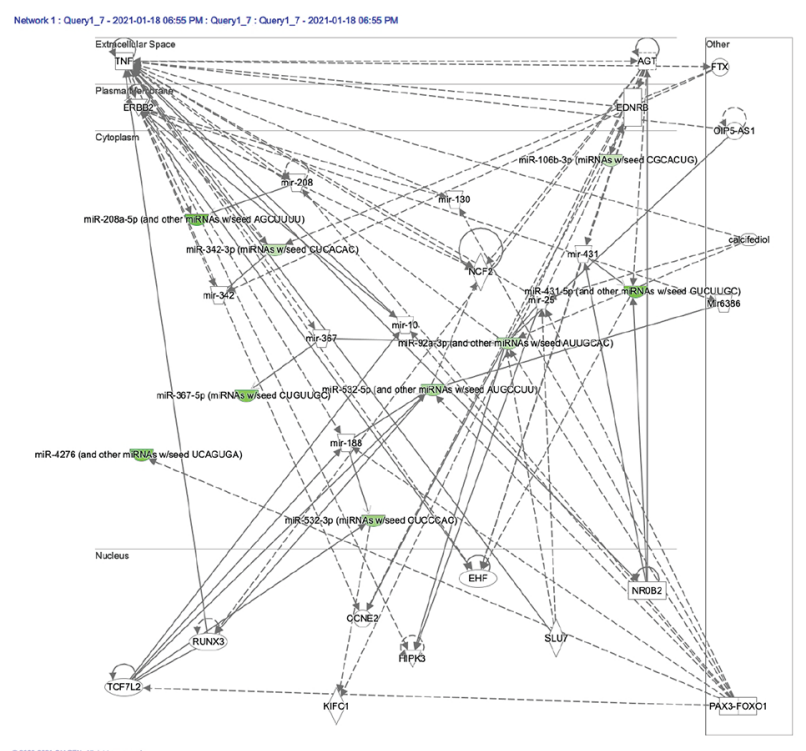

B

MSigDB Hallmark 2020

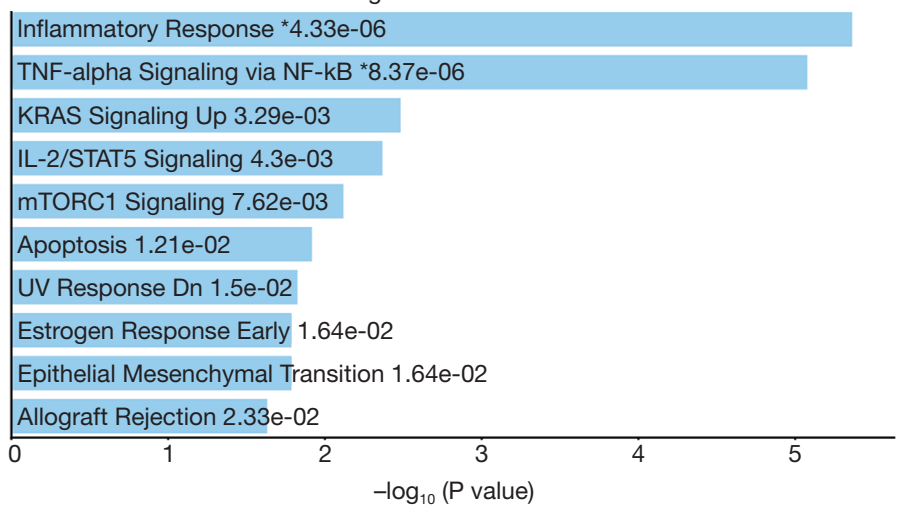

C

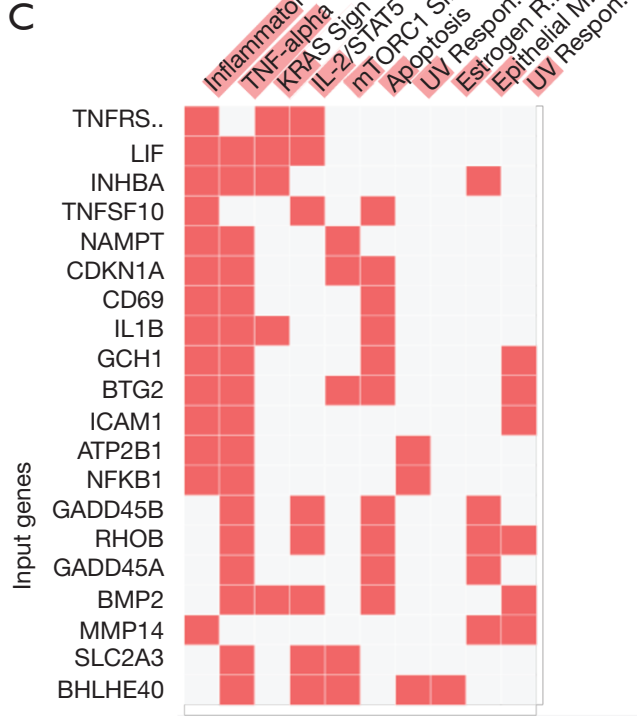

Figure 7 Major target genes of smRNA which expression is low at terminal sedation (purple cluster), including anti-inflammatory genes. (A) Gene network that is controlled by the down-regulated smRNAs (green) in the purple cluster. Cellular locations of the genes targeted by the smRNAs are also denoted. Dotted line and solid line indicate indirect and direct regulations, respectively. (B) Bar graph is showing significantly enriched functional pathway terms of the target genes of smRNAs in the purple cluster. Molecular Signatures Database (MSigDB) Hallmark version 2020 was used as a pathway dataset. (C) Clustergram of the genes that are included in the highly enriched functional pathways in (B). Anti-inflammatory genes are noticeably enriched. 
A
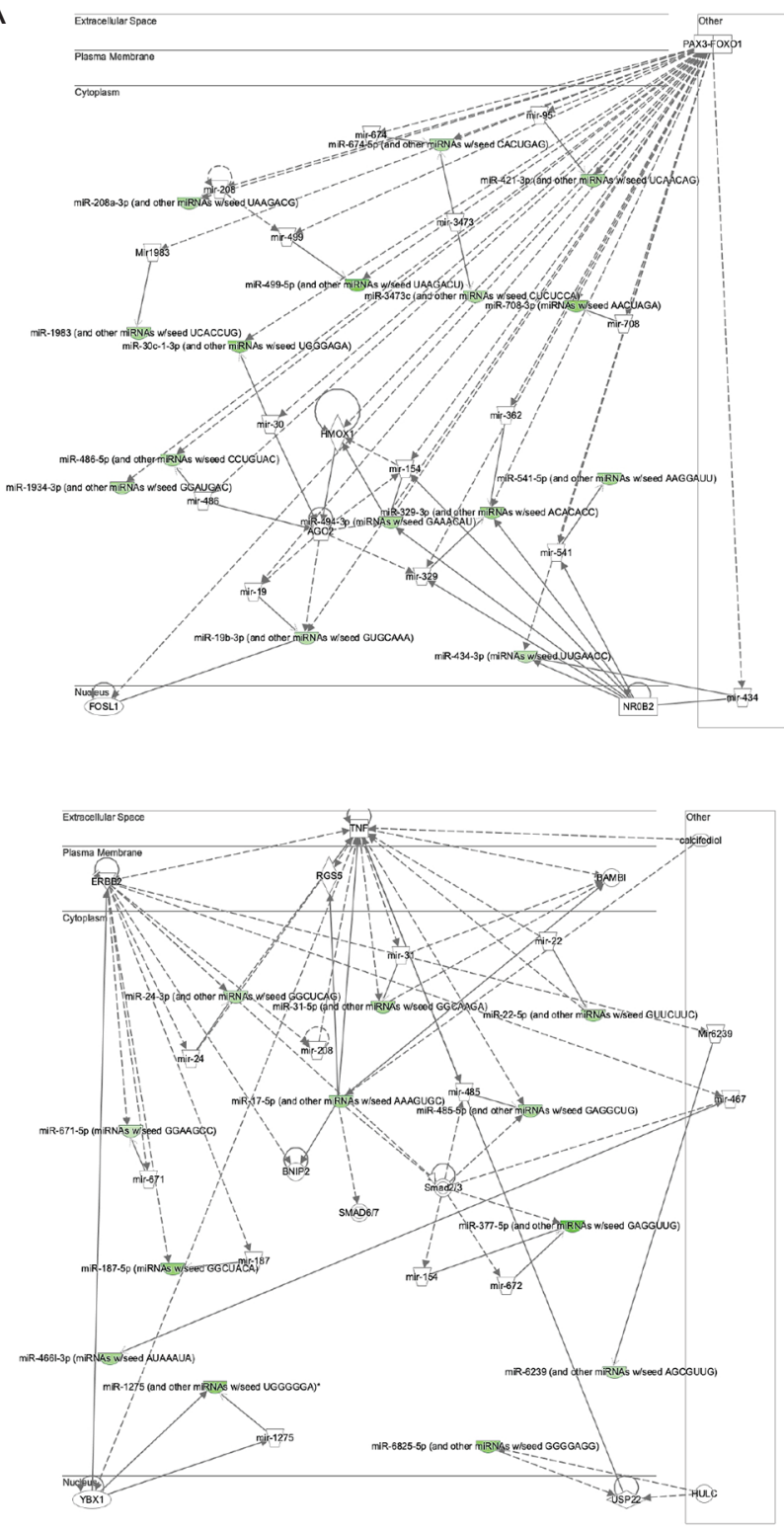

B

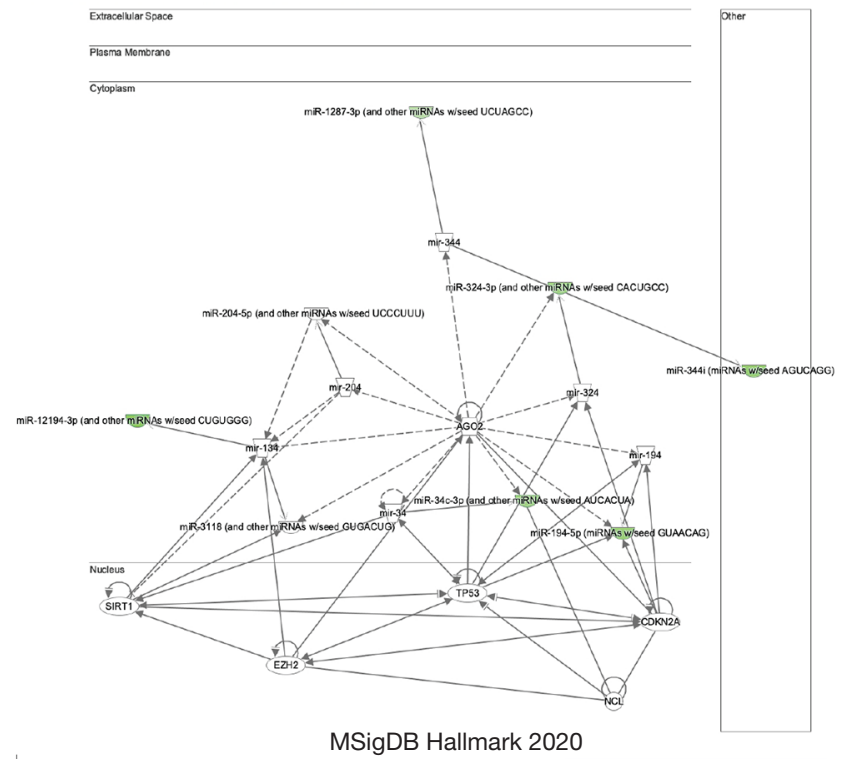

Inflammatory Response *1.14e-07

UV Response Dn *1.27e-06

TNF-alpha Signaling via NF-kB *6.32e-06

IL-6/JAK/STAT3 Signaling *4.29e-05

PI3K/AKT/mTOR Signaling *2.72e-04

Estrogen Response Early *3.15e-04

Epithelial Mesenchymal Transition ${ }^{*} 5.52 \mathrm{e}-04$

Apical Surface * $* .85 \mathrm{e}-03$

Apoptosis *1.98e-03

Hypoxia ${ }^{\star 2} .57 \mathrm{e}-03$

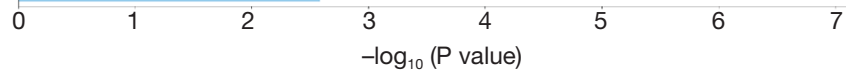

C

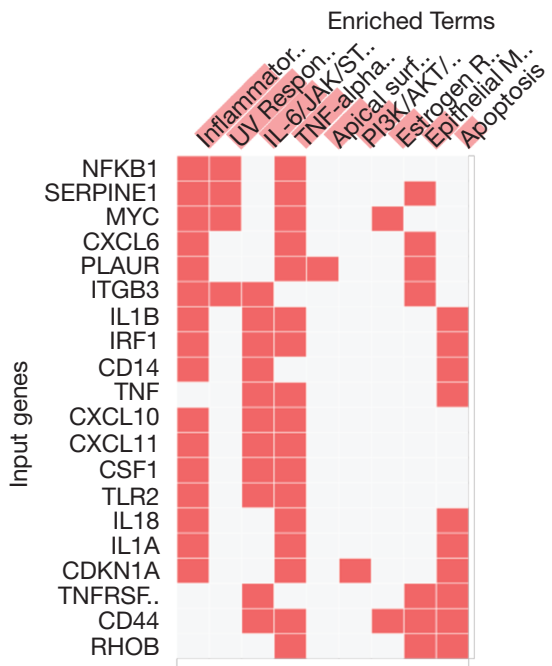

Figure 8 Major target genes of smRNA for which expression is high at 6 weeks before termination but low at terminal sedation (pink cluster), including pro-inflammatory genes. (A) Gene networks that are controlled by the down-regulated smRNAs (green) in the pink cluster. Three networks were predicted from the smRNAs in the pink cluster. (B) Bar graph is showing significantly enriched functional pathway terms of the target genes of smRNAs in the pink cluster. (C) Clustergram of the genes that are included in the highly enriched functional pathways in (B). Pro-inflammatory genes are enriched in this list. 
A

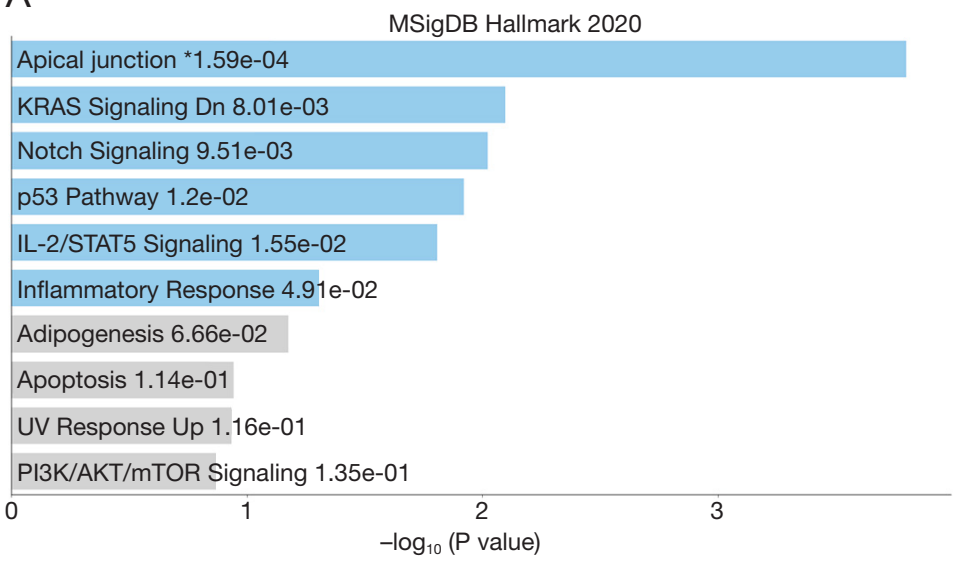

B

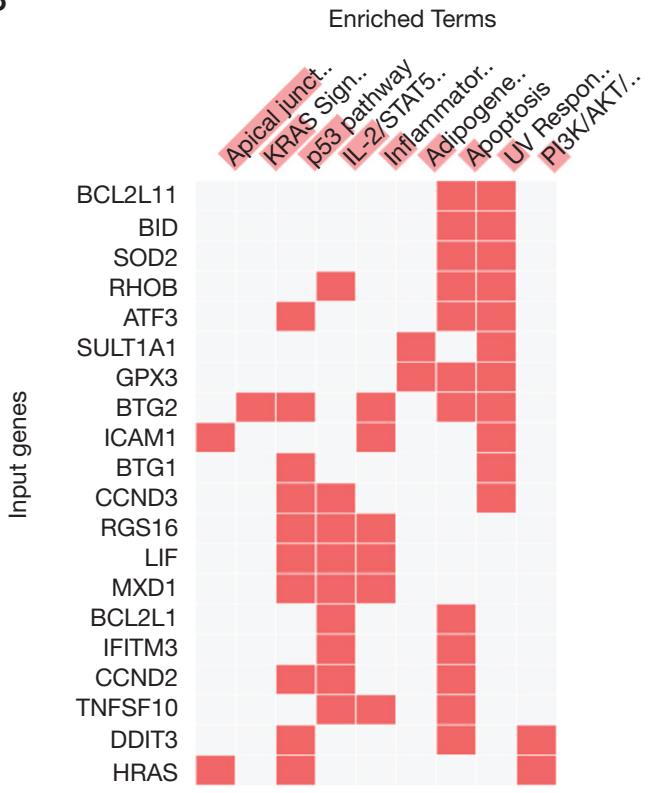

Figure 9 Major target genes of smRNA which expression increases over pathogenesis of IBD. (A) Bar graph is showing significantly enriched functional pathway terms of the target genes of smRNAs in the "cluster13" that has a consistent increase of the expression throughout pathogenesis. Statistically weak pathways are colored in gray. (B) Clustergram of the genes that are included in the highly enriched functional pathways in (A).

development rate may be due to a sex bias, but a clear link has yet to be recorded in human cases $(30,31)$.

The novel fecal smRNA extraction method that we developed produced a robust number of exosomes to use for downstream analysis as shown in both the NanoSight and TapeStation data (Figure 3).

Through analysis of the smRNA data, we identified 504 smRNA that may be implicated in IBD. The timecourse analysis using start-, mid- (6 weeks before endpoint) and end-point datasets revealed 4 significant smRNA clusters classified based on the trend of expression changes over the course of disease progression (Figure 6). Of note, two clusters, named purple and pink clusters, show sharp expression reduction at the end-point when animals were clinically symptomatic. The pink cluster showed an interesting upward trend in the mid-point, when animals were still clinically asymptomatic. Target gene pathway analysis associated with the pink cluster indicated proinflammatory genes are downregulated at the mid-point, when an immediate intervention should be considered although the animals are still asymptomatic. A possible treatment would be administering smRNAs defined in the pink cluster. The expression of the smRNA in the pink cluster is increased at 6 weeks before the end-point (midpoint), however, those expressions then become significantly lower than the start-point. A continuous administration of those smRNA might have a potential in preventing the progression of IBD.

As biomarkers of IBD at an earlier stage of pathogenesis, the smRNAs in the pink cluster and the cluster 13 may be combined to determine the disease progression stage.

\section{Conclusions}

In summary, our study proposes that fecal exosomal smRNA profiling may offer a new opportunity to monitor the inflammatory status of the gut with a capability of detecting its pro-inflammatory (asymptomatic) status. IBD is considered to progress slowly with spatiotemporally dynamic interplay of multiple subsets of immune cells (32). Our next step is to understand from which cells these exosomes are secreted and their physiological impact in vivo. In addition, exosomes are also known to contain other biomolecules, such as mRNA, DNA, protein, and lipids. Multi-omic analysis of the fecal exosome may be required as well to understand the comprehensive makeup of the fecal exosome which can then 
be applied to a development of reengineered exosomes that can be utilized to treat the inflamed colonic lesion (33).

\section{Acknowledgments}

We thank Georgina Bixler, MS, Robert Brucklacher, MS, Emily Demchak, BS, and Teodora Orendovic, PhD of Penn State College of Medicine Genome Sciences Facility for small RNA-sequencing services. We also thank Han Chen, MD, PhD of Penn State College of Medicine Transmission Electron Microscopy Facility for assistance with transmission electron microscopy images, and Jeffrey $M$ Sundstrom, MD, PhD and Yuanjun Zhao, $\mathrm{PhD}$ of Penn State College of Medicine Department of Ophthalmology for assistance with NanoSight data.

Funding: This work was supported by the Laboratory Animal Medicine MS program at Penn State College of Medicine Department of Comparative Medicine.

\section{Footnote}

Reporting Checklist: The authors have completed the ARRIVE reporting checklist. Available at https://dx.doi. org/10.21037/exrna-21-8

Data Sharing Statement: All raw data and processed data of RNA sequencing are available (GSE167239). The datasets generated during the study are available from the corresponding author on reasonable request. Available at https://dx.doi.org/10.21037/exrna-21-8

Conflicts of Interest: All authors have completed the ICMJE uniform disclosure form (available at https://dx.doi. org/10.21037/exrna-21-8). The authors have no conflicts of interest to declare.

Ethical Statement: The authors are accountable for all aspects of the work in ensuring that questions related to the accuracy or integrity of any part of the work are appropriately investigated and resolved. All experiments were conducted in accordance with institutional guidelines, the Guide for the Care and Use of Laboratory Animals (Institute for Laboratory Animal Research, 2011) and approved by the Penn State College of Medicine Institutional Animal Care and Uses Committee (ID: PROTO201800170).

Open Access Statement: This is an Open Access article distributed in accordance with the Creative Commons Attribution-NonCommercial-NoDerivs 4.0 International License (CC BY-NC-ND 4.0), which permits the noncommercial replication and distribution of the article with the strict proviso that no changes or edits are made and the original work is properly cited (including links to both the formal publication through the relevant DOI and the license). See: https://creativecommons.org/licenses/by-nc-nd/4.0/.

\section{References}

1. Hanauer SB. Inflammatory bowel disease: epidemiology, pathogenesis, and therapeutic opportunities. Inflamm Bowel Dis 2006;12 Suppl 1:S3-9.

2. Schaefer JS. MicroRNAs: how many in inflammatory bowel disease? Curr Opin Gastroenterol 2016;32:258-66.

3. Baumgart DC, Sandborn WJ. Inflammatory bowel disease: clinical aspects and established and evolving therapies. Lancet 2007;369:1641-57.

4. Bewtra M, Su C, Lewis JD. Trends in hospitalization rates for inflammatory bowel disease in the United States. Clin Gastroenterol Hepatol 2007;5:597-601.

5. Dahlhamer JM, Zammitti EP, Ward BW, et al. Prevalence of Inflammatory Bowel Disease Among Adults Aged $\geq 18$ Years - United States, 2015. MMWR Morb Mortal Wkly Rep 2016;65:1166-9.

6. Maaser C, Sturm A, Vavricka SR, et al. ECCO-ESGAR Guideline for Diagnostic Assessment in IBD Part 1: Initial diagnosis, monitoring of known IBD, detection of complications. J Crohns Colitis 2019;13:144-64.

7. Bernstein CN, Fried M, Krabshuis JH, et al. World Gastroenterology Organization Practice Guidelines for the diagnosis and management of IBD in 2010. Inflamm Bowel Dis 2010;16:112-24.

8. Cantoro L, Di Sabatino A, Papi C, et al. The Time Course of Diagnostic Delay in Inflammatory Bowel Disease Over the Last Sixty Years: An Italian Multicentre Study. J Crohns Colitis 2017;11:975-80.

9. Lee DW, Koo JS, Choe JW, et al. Diagnostic delay in inflammatory bowel disease increases the risk of intestinal surgery. World J Gastroenterol 2017;23:6474-81.

10. Wani S, Man Law IK, Pothoulakis C. Role and mechanisms of exosomal miRNAs in IBD pathophysiology. Am J Physiol Gastrointest Liver Physiol 2020;319:G646-54.

11. Zhou B, Xu K, Zheng X, et al. Application of exosomes as liquid biopsy in clinical diagnosis. Signal Transduct Target 
Ther 2020;5:144.

12. Bhat NS. Dar AA, Saini S, et al. Abstract 1111: Role of a prometastatic miRNA as a negative regulator of the key metastasis suppressor genes in renal cell carcinoma 2018; American Association for Cancer Research 2018.

13. Chen Y, Gao C, Sun Q, et al. MicroRNA-4639 Is a Regulator of DJ-1 Expression and a Potential Early Diagnostic Marker for Parkinson's Disease. Front Aging Neurosci 2017;9:232.

14. Xiong DD, Lv J, Wei KL, et al. A nine-miRNA signature as a potential diagnostic marker for breast carcinoma: An integrated study of 1,110 cases. Oncol Rep 2017;37:3297-304.

15. Viennois E, Zhao Y, Han MK, et al. Serum miRNA signature diagnoses and discriminates murine colitis subtypes and predicts ulcerative colitis in humans. Sci Rep 2017;7:2520.

16. Koga Y, Yasunaga M, Moriya Y, et al. Exosome can prevent RNase from degrading microRNA in feces. J Gastrointest Oncol 2011;2:215-22.

17. Liu S, da Cunha AP, Rezende RM, et al. The Host Shapes the Gut Microbiota via Fecal MicroRNA. Cell Host Microbe 2016;19:32-43.

18. Cominelli F, Arseneau KO, Rodriguez-Palacios A, et al. Uncovering Pathogenic Mechanisms of Inflammatory Bowel Disease Using Mouse Models of Crohn's DiseaseLike Ileitis: What is the Right Model? Cell Mol Gastroenterol Hepatol 2017;4:19-32.

19. Dieleman LA, Arends A, Tonkonogy SL, et al. Helicobacter hepaticus does not induce or potentiate colitis in interleukin-10-deficient mice. Infect Immun 2000;68:5107-13.

20. Kühn R, Löhler J, Rennick D, et al. Interleukin-10deficient mice develop chronic enterocolitis. Cell 1993;75:263-74.

21. Rennick DM, Fort MM, Davidson NJ. Studies with IL10-/- mice: an overview. J Leukoc Biol 1997;61:389-96.

22. Scheinin T, Butler DM, Salway F, et al. Validation of the interleukin-10 knockout mouse model of colitis:

doi: 10.21037/exrna-21-8

Cite this article as: Manning S, Torii S, Atkins HM, Kawasawa YI. Dynamic expression of smRNA from fecal exosome in disease progression of an inflammatory bowel disorder mouse model. ExRNA 2021;3:2. antitumour necrosis factor-antibodies suppress the progression of colitis. Clin Exp Immunol 2003;133:38-43.

23. The Jackson Laboratories. Available online: https://www. jax.org/strain/002251

24. Sharma P, Aguilar R, Siddiqui OA, et al. Secondary systemic amyloidosis in inflammatory bowel disease: a nationwide analysis. Ann Gastroenterol 2017;30:504-11.

25. Stidham RW, Higgins PDR. Colorectal Cancer in Inflammatory Bowel Disease. Clin Colon Rectal Surg 2018;31:168-78.

26. Erben U, Loddenkemper C, Doerfel K, et al. A guide to histomorphological evaluation of intestinal inflammation in mouse models. Int J Clin Exp Pathol 2014;7:4557-76.

27. Jiménez-Jacinto V, Sanchez-Flores A, Vega-Alvarado L. Integrative Differential Expression Analysis for Multiple EXperiments (IDEAMEX): A Web Server Tool for Integrated RNA-Seq Data Analysis. Front Genet 2019;10:279.

28. Ernst J, Bar-Joseph Z. STEM: a tool for the analysis of short time series gene expression data. BMC Bioinformatics 2006;7:191.

29. Kuleshov MV, Jones MR, Rouillard AD, et al. Enrichr: a comprehensive gene set enrichment analysis web server 2016 update. Nucleic Acids Res 2016;44:W90-7.

30. Rosenblatt E, Kane S. Sex-Specific Issues in Inflammatory Bowel Disease. Gastroenterol Hepatol (N Y) 2015;11:592-601.

31. Shah SC, Khalili H, Gower-Rousseau C, et al. Sex-Based Differences in Incidence of Inflammatory Bowel DiseasesPooled Analysis of Population-Based Studies From Western Countries. Gastroenterology 2018;155:10791089.e3.

32. Koscsó B, Kurapati S, Rodrigues RR, et al. Gut-resident CX3CR1hi macrophages induce tertiary lymphoid structures and IgA response in situ. Sci Immunol 2020;5:eaax0062.

33. Zhang H, Wang L, Li C, et al. Exosome-Induced Regulation in Inflammatory Bowel Disease. Front Immunol 2019;10:1464. 


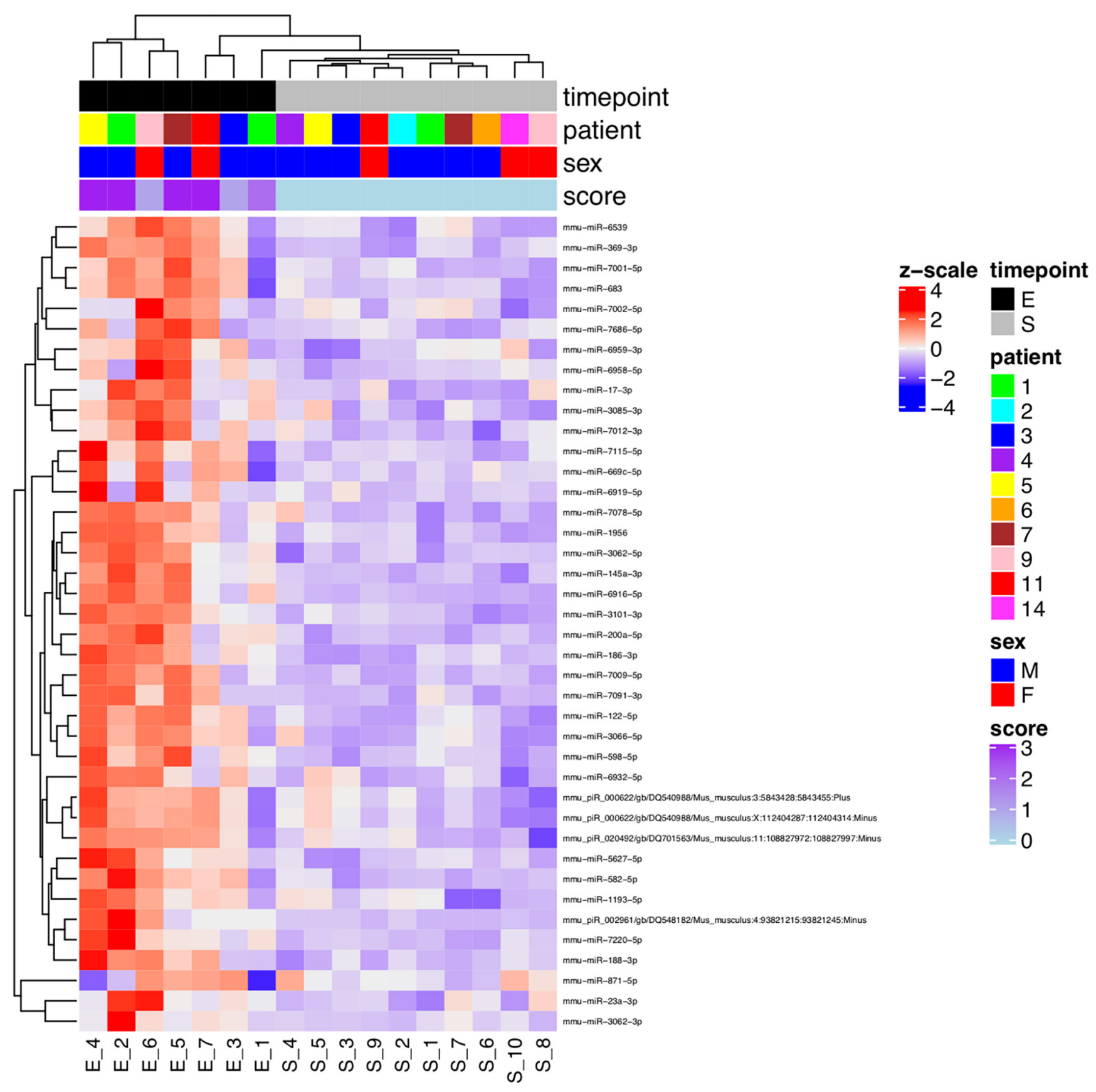

Figure S1 Differentially expressed genes between before symptom onset and terminal sedation. A clustered heatmap of the 40 differentially expressed smRNAs that are consistently called significantly different among 4 algorithms, edgeR, DESeq2, NOI-seq, and limma-voom. The annotation bars depict sample information for its timepoint, mouse identity (patient), sex, and disease scores when feces were sampled, respectively. 


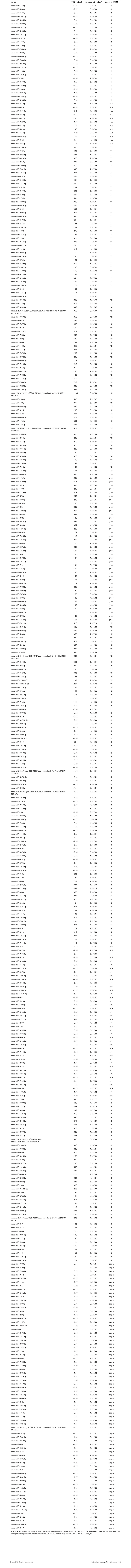

